\title{
The impact of comorbid psychiatric disorders on methadone maintenance treatment in opioid use disorder: a prospective cohort study
}

This article was published in the following Dove Press journal:

Neuropsychiatric Disease and Treatment

24 May 2017

Number of times this article has been viewed

\author{
Tea Rosic' \\ Leen $\mathrm{Naji}^{2}$ \\ Monica Bawor ${ }^{3}$ \\ Brittany B Dennis ${ }^{3}$ \\ Carolyn Plater ${ }^{4}$ \\ David C Marsh ${ }^{5}$ \\ Lehana Thabane ${ }^{6-8}$ \\ Zainab Samaan ${ }^{6-11}$
}

'St Joseph's Healthcare, ${ }^{2}$ Michael G DeGroote School of Medicine, McMaster University, Hamilton, ON, Canada; ${ }^{3}$ St George's University of London, London, UK; ${ }^{4}$ Canadian Addiction Treatment Centre,

Richmond Hill, ${ }^{5}$ Northern Ontario

School of Medicine, Sudbury,

${ }^{6}$ Biostatistics Unit, Research Institute,

St Joseph's Healthcare, ${ }^{7}$ Department

of Clinical Epidemiology and

Biostatistics, McMaster University,

${ }^{8}$ Peter Boris Centre for Addictions

Research, ${ }^{9}$ Mood Disorders Research

Unit, St Joseph's Healthcare,

${ }^{10}$ Population Genomics Program, Chanchlani Research Centre,

"Department of Psychiatry and Behavioural Neurosciences, McMaster University, Hamilton, ON, Canada
Correspondence: Zainab Samaan Mood Disorders Program, St Joseph's Healthcare Hamilton, 100 West 5th Street, Hamilton, ON L8N 3K7, Canada $\mathrm{Tel}+\mathrm{I} 905522$ II 55 ext 35448 Fax + I $90538 \mid 5629$ Email samaanz@mcmaster.ca
Objective: There is a significant interindividual variability in treatment outcomes in methadone maintenance treatment (MMT) for opioid use disorder (OUD). This prospective cohort study examines the impact of comorbid psychiatric disorders on continued illicit opioid use in patients receiving MMT for OUD.

Methods: Data were collected from 935 patients receiving MMT in outpatient clinics between June 2011 and June 2015. Using linear regression analysis, we evaluated the impact of having a comorbid psychiatric disorder on continued illicit opioid use during MMT, adjusting for important confounders. The main outcome measure was percentage of opioid-positive urine screens for 6 months. We conducted a subgroup analysis to determine the influence of specific comorbid psychiatric disorders, including substance use disorders, on continued illicit opioid use.

Results: Approximately $80 \%$ of participants had at least one comorbid psychiatric disorder in addition to OUD, and $42 \%$ of participants had a comorbid substance use disorder. There was no significant association between having a psychiatric comorbidity and continuing opioid use $(P=0.248)$. Results from subgroup analysis, however, suggest that comorbid tranquilizer ( $\beta=20.781, P<0.001)$ and cocaine $(\beta=6.344, P=0.031)$ use disorders are associated with increased rates of continuing opioid use.

Conclusion: Results from our study may serve to guide future MMT guidelines. Specifically, we find that cocaine or tranquilizer use disorder, comorbid with OUD, places patients at high risk for poor MMT outcomes. Treatment centers may choose to gear more intensive therapy toward such populations.

Keywords: opioid use disorder, methadone, substance abuse, comorbidity, psychiatric disorder

\section{Introduction}

Opioid use disorder (OUD) is the largest contributor to disability-adjusted life years caused by drug use worldwide. ${ }^{1}$ Efforts to maximize treatment efficacy and reduce opioid abuse are timely as the prevalence of OUD is on the rise, ${ }^{2,3}$ with its global burden increasing by $74 \%$ between 1990 and $2010 .^{4}$

Methadone maintenance treatment (MMT) is a widely implemented and effective treatment for OUD, ${ }^{5}$ yet there is significant interindividual variability in treatment effectiveness and outcomes. ${ }^{6-8}$ To improve treatment, research groups are actively investigating the factors that influence MMT outcomes. ${ }^{9-15}$ Well-documented predictors of treatment outcome include length of time in treatment, ${ }^{11-13}$ methadone dose,${ }^{14}$ and injecting behavior. ${ }^{15}$ The impact of comorbid psychiatric disorders on MMT outcome, however, remains unclear. 
Comorbid psychiatric disorders place individuals using recreational or prescription opioids at an increased risk for developing OUD. ${ }^{16-18}$ Similarly, patients with OUD have a higher lifetime prevalence of psychiatric disorders, including other substance use disorders (SUDs), compared to that in the general population. ${ }^{19,20}$ As psychiatric comorbidity is associated with increased risk for opioid dependence, one may infer that it similarly impacts MMT outcomes. ${ }^{21-30}$ However, current literature investigating the relationship between psychiatric comorbidity and MMT outcomes is inconclusive, with some studies reporting lack of association, ${ }^{21-24}$ while others document adverse impact ${ }^{25-28}$ or protective effects of psychiatric comorbidity. ${ }^{29-30}$

In this prospective cohort study, we investigated the influence of comorbid psychiatric disorders on MMT outcome during a 6-month duration in patients with OUD. Our objective was to determine the impact of a patient's psychiatric profile (ie, the presence of comorbid psychiatric disorders and use of psychotropic medications) on response to MMT treatment.

Specifically, we examined the following:

1. The prevalence of comorbid psychiatric disorders in a cohort of patients with OUD receiving MMT.

2. The impact of comorbid psychiatric disorders on continued opioid use during MMT as assessed by urine drug screen.

3. The association between specific comorbid psychiatric and SUDs and continued opioid use during MMT.

\section{Methods}

We used observational data from the Genetics of Opioid Addiction (GENOA) program in this study. ${ }^{31}$ We collected data from patients with OUD receiving MMT at 13 community-based outpatient methadone clinics across Southern Ontario, Canada, between June 2011 and June 2015. Ethics approval was obtained from the Hamilton Integrated Research Ethics Board (project ID 11-056), and written informed consent was obtained from each study participant. Further details related to GENOA study methods have been previously reported. ${ }^{31-34}$ This study is reported in accordance with the Strengthening the Reporting of Observational studies in Epidemiology (STROBE) guidelines. ${ }^{35}$

\section{Participants}

Using the following inclusion criteria, we screened and recruited study participants: males and females aged 18 years or older, diagnosed with OUD as per the Diagnostic and Statistical Manual of Mental Disorders, Fourth Edition $(\mathrm{DSM}-\mathrm{IV})^{36}$ criteria, and receiving MMT for their OUD.
Participants must have also been able to provide written informed consent.

Participants receiving opioid substitution therapy other than methadone were excluded from the study, as were those unable to communicate in English. All treatment centers included in our study are managed centrally and follow the same management protocols.

\section{Data collection and instruments}

Participants provided information on sociodemographic factors, medical history, current medications, daily methadone dose, and length of time in treatment through face-to-face interviews. Participants also completed the Mini-International Neuropsychiatric Interview (MINI) version 6.0, ${ }^{37}$ administered by trained interviewers. The MINI has been validated against both the Structured Clinical Interview for DSM diagnoses (SCID) ${ }^{38}$ and the Composite International Diagnostic Interview for ICD-10 (CIDI) ${ }^{39}$ in English. ${ }^{37}$

We administered all modules of the MINI 6.0, including mood disorder (major depression or bipolar affective disorder), anxiety disorder (generalized anxiety disorder, social phobia, posttraumatic stress disorder, obsessive compulsive disorder, and panic disorder with and without agoraphobia), psychotic disorder (schizophrenia, schizoaffective disorder, brief psychotic disorder, delusional disorder, substance-induced psychosis, and psychosis not otherwise specified [NOS]), antisocial personality disorder, eating disorder (anorexia nervosa and bulimia nervosa), alcohol use disorder, and SUD. ${ }^{37}$

MINI diagnoses of anxiety disorders and eating disorders reflect only current symptomatology in the past month, whereas diagnoses of mood, psychotic, and antisocial personality disorders reflect past year and lifetime symptomatology. ${ }^{37}$ Diagnoses of alcohol and substance abuse reflect 12-month history of symptomatology. ${ }^{37}$ Substances assessed for abuse in the MINI include stimulants, cocaine, narcotics, hallucinogens, phencyclidine, inhalants, cannabis, tranquilizers, and "other" (ie, steroids, nonprescription sleep or diet pills, and cough medicine). ${ }^{37}$

\section{Urine toxicology analysis}

In MMT clinical sites, methadone is provided to patients under supervision, and urine drug screens for illicit opioids are conducted at weekly or biweekly frequency. We collected information on illicit opioid use patterns through urine toxicology screening, using the iMDx ${ }^{\mathrm{TM}}$ Prep assay. ${ }^{40}$ This assay detects opioids present in the urine at concentrations above $300 \mathrm{ng} / \mathrm{mL}$ and distinguishes between methadone, prescribed synthetic opioids, and naturally occurring opioids. ${ }^{40}$ 
For the purpose of this study, an "opioid-positive" urine drug screen is defined by the presence of a nonmethadone opioid in a patient's urine sample, with the exception of those receiving prescription opioids for reasons other than OUD (eg, chronic pain), confirmed by their documented medical chart.

\section{Primary analysis and outcome measures}

Our objective is to determine the association between presence of psychiatric comorbidity and MMT outcome. The primary outcome in this study is continued illicit opioid abuse during a 6-month period of MMT, as detected by opioidpositive urine samples. Results are measured as the percentage of opioid-positive urine drug screens per total number of urine screens available over a 6-month duration.

Although we acknowledge that MMT success may be assessed through several treatment outcomes, including retention in treatment, risk taking behaviors, and social stability, the primary indication of MMT is to ameliorate withdrawal symptoms, reduce cravings for opioids, and ultimately promote abstinence from illicit opioids. ${ }^{41,42}$ Urine toxicology is the gold standard for detecting illicit opioid use during MMT, and many studies have used the absence of illicit opioids, as measured through urine drug screens, as a treatment outcome indicator for MMT. ${ }^{41,43}$

We compare demographic and clinical information of patients with psychiatric comorbidity, as diagnosed by MINI, to those with no comorbid psychiatric diagnoses. For the purposes of this study, we define "psychiatric comorbidity" as the presence of any MINI diagnosis in addition to OUD.

We constructed a multiple linear regression model, in which percentage of opioid-positive urine drug screens was the continuous-dependent variable. Covariates included psychiatric comorbidity (a bivariate variable, noting presence, or absence of any MINI diagnosis), age, sex, methadone dose, duration in MMT, and psychotropic medications. Results are reported in $\beta$ coefficient and standard error. Positive $\beta$ values indicate a higher percentage of opioid-positive urine drug screens and thus higher illicit opioid use.

To further assess the relationship between psychiatric comorbidity and MMT outcome, we conducted a sensitivity analysis in which we used the multiple linear regression model described above but excluded participants who did not have a MINI-diagnosed psychiatric comorbidity yet were treated with psychotropic medications $(n=51)$ to avoid missing cases of psychiatric comorbidity.

\section{Secondary analyses}

We conducted a subgroup analysis to determine the association between specific comorbid psychiatric and SUDs and continued illicit opioid use. We used a multiple linear regression model in which percentage of opioid-positive urine drug screens was the dependent variable. Covariates included age, sex, methadone dose, and duration in MMT, as well as the following aforementioned psychiatric disorder groups: mood disorders, anxiety disorders, psychotic disorders, antisocial personality disorder, and alcohol use disorder. Due to low prevalence of eating disorders $(n=7)$, we excluded eating disorders from our regression analyses. We also included comorbid SUD variables, grouped by the substance used: stimulants, cocaine, hallucinogens, phencyclidines, cannabis, tranquilizers, and other.

\section{Statistical analysis}

All statistical analyses were performed using STATA version 12.1 (StataCorp LP, College Station, TX, USA). We present patient demographic data by psychiatric comorbidity status, assessed using independent sample $t$-tests and Chi-square tests. Mean values and standard deviations (SDs) are reported for continuous variables, and percentages for categorical variables. Multivariable linear regression analyses were conducted as described above. We assessed for multicollinearity using variance inflation factor testing. The level of significance for hypothesis testing was set at $\alpha=0.05$. After excluding 279 participants for not having MINI or urine drug screen data, we had 652 participants included in the analysis (Figure 1, participants flow diagram). Our sample size of 652 participants was adequately powered to perform these analyses allowing for more than 10 participants per covariate. ${ }^{44}$

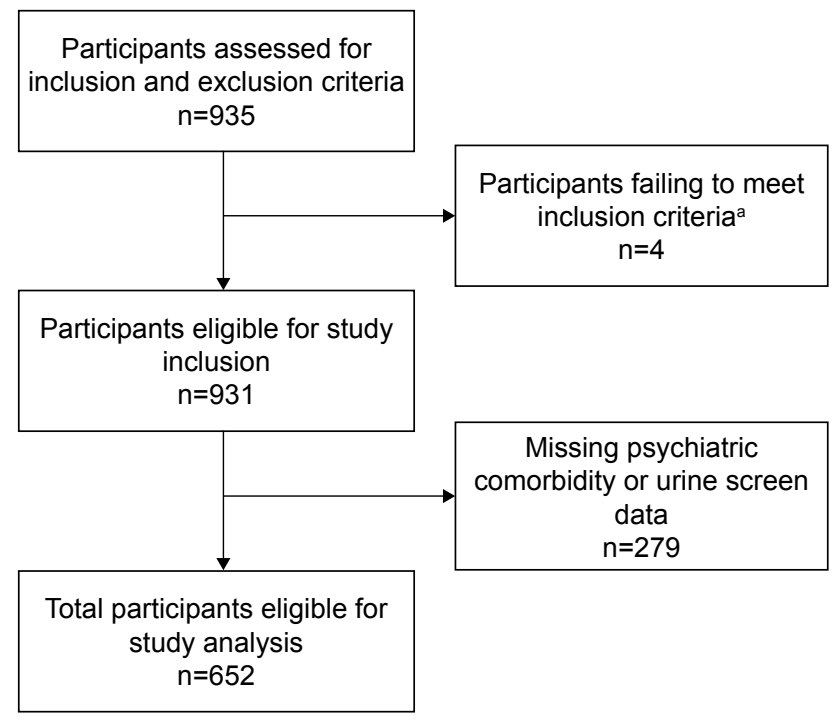

Figure I GENOA study eligibility and screening of participants.

Note: axcluded due to treatment with buprenorphine for substitute opioid therapy. Abbreviation: GENOA, Genetics of Opioid Addiction. 


\section{Results}

\section{Characteristics of participants with and without psychiatric comorbidity}

Our sample consisted of $78.53 \%$ of participants with a comorbid psychiatric disorder $(n=512)$, of which $48.44 \%$ were females, with a mean age of 37.96 years (SD: 10.70; Table 1).

Participants with psychiatric comorbidity were younger $(P<0.001)$, more commonly males $(P=0.012)$, and more likely to be unemployed $(P=0.005)$. Participants with psychiatric comorbidity were also more commonly using a psychotropic medication $(P=0.01)$. The mean proportion of opioid-positive urine drug screens obtained from participants with psychiatric comorbidity was $18.93 \%$ (SD: 27.42), compared to $17 \%$ (SD: 27.18 ) in those without a psychiatric comorbidity, which was not significantly different $(P=0.46$; Table 1). In the psychiatric comorbidity group, $32.42 \%$ of participants had zero opioid-positive urine drug screens, as compared to $37.86 \%$ of participants without psychiatric comorbidity $(P=0.228$; Table 1$)$.

\section{Prevalence of psychiatric comorbidity and use of psychotropic medication}

Anxiety disorders were the most common comorbid psychiatric disorder identified, with $42.64 \%$ of participants meeting MINI diagnostic criteria for generalized anxiety disorder, social phobia, posttraumatic stress disorder, obsessive-compulsive disorder, or panic disorder (Table 2). We identified mood disorders in $41.41 \%$ of participants, and comorbid mood and anxiety disorders in $20.12 \%$ of participants (Table 2).

A comorbid SUD, in addition to OUD, was identified in $41.56 \%$ of participants. The most commonly abused substance was cannabis (27.91\%) followed by cocaine (19.94\%) and tranquilizers (4.29\%; Table 2).

Just under half $(46.01 \%)$ of the participants were prescribed psychotropic medications in addition to methadone (Table $1 ; n=300$ ), with $24.54 \%$ reporting the use of two or more psychotropic medications (data not shown). Antidepressants were the most commonly used psychotropic medication $(27.45 \%)$, followed by benzodiazepines (23.93\%), antipsychotics (12.73\%), stimulants (4.14\%), nonbenzodiazepine sedatives $(2.91 \%)$, and mood stabilizers (1.84\%; data not shown). Participants with psychiatric comorbidity were more commonly taking antidepressants and antipsychotics (data not shown). There was no significant difference between the two groups in their use of benzodiazepines, nonbenzodiazepine sedatives, stimulants, or mood stabilizers (data not shown).

\section{Impact of psychiatric comorbidity on MMT outcome}

Our primary analysis did not show a significant association between presence of a psychiatric comorbidity and

Table I Characteristics of participants with and without psychiatric comorbidity receiving methadone treatment

\begin{tabular}{|c|c|c|c|}
\hline Characteristics, $\mathbf{N}=652$ & $\begin{array}{l}\text { Psychiatric comorbidity, } \\
n=512\end{array}$ & $\begin{array}{l}\text { No psychiatric } \\
\text { comorbidity, } n=\mid 40\end{array}$ & P-value \\
\hline Current age (years), mean \pm SD (range) & $37.96 \pm 10.70(18-65)$ & $42.49 \pm 11.67(21-64)$ & $<0.001$ \\
\hline \multirow[t]{2}{*}{ Sex, $\mathrm{n}$ (\% of subgroup) } & M: $264(51.56)$ & M: $89(63.57)$ & 0.012 \\
\hline & F: 248 (48.44) & F: $5 \mathrm{I}(36.43)$ & \\
\hline \multirow[t]{6}{*}{ Highest completed education, $n$ (\% of subgroup) } & None: I $(0.20)$ & None: 0 & 0.357 \\
\hline & Elementary: 95 (18.63) & Elementary: I6 (I I.59) & \\
\hline & Secondary: 27I (53.14) & Secondary: 85 (6I.59) & \\
\hline & Trade school: 21 (4.12) & Trade school: 7 (5.07) & \\
\hline & College/university: I2I (23.73) & College/university: 30 (2I.74) & \\
\hline & Masters/PhD: I (0.20) & Masters/PhD: 0 & \\
\hline Unemployment, $\mathrm{n}$ (\% of subgroup) & $350(68.36)$ & $78(55.7 \mathrm{I})$ & 0.005 \\
\hline Using psychotropic medication, ${ }^{\mathrm{b}} \mathrm{n}$ (\% of subgroup) & $249(48.63)$ & $5 I(36.43)$ & 0.010 \\
\hline Methadone dose $(\mathrm{mg} / \mathrm{d})$, mean $\pm \mathrm{SD}$ (range) & $77.12 \pm 45.84(2-400)$ & $78.67 \pm 48.39(6-280)$ & 0.726 \\
\hline Duration on MMT (months), mean \pm SD (range) & $50.18 \pm 50.87(0.1-276)$ & $52.70 \pm 46.22(0.3-216)$ & 0.597 \\
\hline Number of urine drug screens completed, ${ }^{\mathrm{c}}$ mean \pm SD (range) & $34.09 \pm 11.98(7-76)$ & $30.75 \pm 11.45(2-74)$ & 0.003 \\
\hline Percent opioid-positive urine drug screens, ${ }^{d}$ mean \pm SD (range) & $18.93 \pm 27.42(0-100)$ & $17.00 \pm 27.18(0-100)$ & 0.460 \\
\hline Abstinence from illicit opioid use, ${ }^{\mathrm{e}} \mathrm{n}$ (\% of subgroup) & $166(32.42)$ & $53(37.86)$ & 0.228 \\
\hline
\end{tabular}

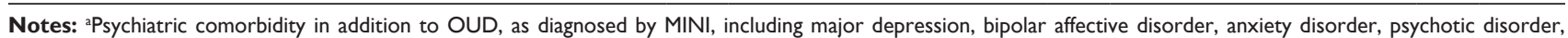

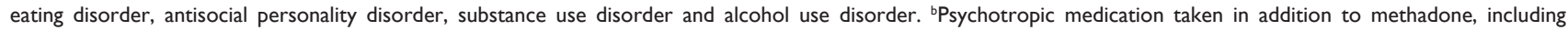
antidepressants, benzodiazepines, nonbenzodiazepine sedatives, antipsychotics, mood stabilizers, and stimulants. 'Number of urine drug screens completed over 6-month period, including 3 months prior to study entry and 3 months poststudy entry. ${ }^{d P e r c e n t a g e ~ o f ~ u r i n e ~ d r u g ~ s c r e e n s ~ d e t e c t i n g ~ i l l i c i t ~ o p i o i d ~ u s e, ~ t a k e n ~ o v e r ~ 6-m o n t h ~ p e r i o d, ~}$

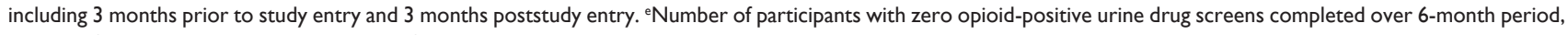
including 3 months prior to study entry and 3 months poststudy entry.

Abbreviations: F, female; M, male; MINI, Mini-International Neuropsychiatric Interview; MMT, methadone maintenance treatment; OUD, opioid use disorder; SD, standard deviation. 
Table 2 Psychiatric comorbidity identified in participants receiving methadone treatment $\mathrm{t}^{\mathrm{a}, \mathrm{b}}$

\begin{tabular}{|c|c|}
\hline Diagnosis & $\begin{array}{l}\text { Number (\% total study } \\
\text { sample), } N=652\end{array}$ \\
\hline Mood disorder ${ }^{c}$ & $270(4 I .4 I)$ \\
\hline Major depression & $184(28.22)$ \\
\hline Bipolar affective disorder type I & $73(11.20)$ \\
\hline Bipolar affective disorder type II & $13(1.99)$ \\
\hline Anxiety disorder ${ }^{d}$ & $278(42.64)$ \\
\hline Generalized anxiety disorder & $128(19.63)$ \\
\hline Social phobia & $94(14.42)$ \\
\hline Posttraumatic stress disorder & 71 (I0.89) \\
\hline Obsessive compulsive disorder & $70(10.74)$ \\
\hline Panic disorder with agoraphobia & $98(15.03)$ \\
\hline Panic disorder without agoraphobia & $30(4.60)$ \\
\hline Co-occurring mood and anxiety disorders & $150(23.01)$ \\
\hline Psychotic disordere & $20(3.07)$ \\
\hline Schizophrenia & $8(1.23)$ \\
\hline Schizoaffective disorder & $0(0)$ \\
\hline Brief psychotic disorder & $\mathrm{I}(0.15)$ \\
\hline Delusional disorder & $6(0.92)$ \\
\hline Substance-induced psychosis & $4(0.61)$ \\
\hline Psychosis NOS & $3(0.46)$ \\
\hline Eating disorder & $7(1.07)$ \\
\hline Anorexia nervosa & $\mathrm{I}(0.17)$ \\
\hline Bulimia nervosa & $6(1.15)$ \\
\hline Antisocial personality disorder & $146(22.39)$ \\
\hline Substance use disorder ${ }^{g}$ & $27 \mid(4 I .56)$ \\
\hline Stimulants & $44(6.75)$ \\
\hline Cocaine & $130(19.94)$ \\
\hline Hallucinogens & $26(3.99)$ \\
\hline Phencyclidines & $6(0.92)$ \\
\hline Inhalants & $0(0)$ \\
\hline Cannabis & I $82(27.9 \mid)$ \\
\hline Tranquilizers & $28(4.29)$ \\
\hline Other & $3(0.46)$ \\
\hline Alcohol use disorder ${ }^{h}$ & $83(12.73)$ \\
\hline
\end{tabular}

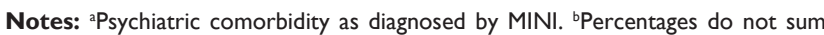
to $100 \%$ as some individuals are diagnosed with multiple comorbid disorders. 'Mood disorder includes major depressive episode, major depressive disorder, and bipolar affective disorder types I and II. 'Anxiety disorder includes generalized anxiety disorder, social phobia, posttraumatic stress disorder, obsessive compulsive disorder, and panic disorder with and without agoraphobia. ePsychotic disorder includes schizophrenia, schizoaffective disorder, brief psychotic disorder, delusiona disorder, substance-induced psychosis, and psychosis NOS. 'Eating disorder includes anorexia nervosa and bulimia nervosa. ${ }^{8}$ Substance use disorder includes substance abuse and substance dependence associated with use of the following substances: stimulants, cocaine, hallucinogens, phencyclidines, inhalants, cannabis, tranquilizers, and other. ${ }^{\mathrm{h}}$ Alcohol use disorder includes alcohol abuse and alcohol dependence.

Abbreviations: MINI, Mini-International Neuropsychiatric Interview; NOS, not otherwise specified.

continuing illicit opioid use ( $P=0.248$; Table 3$)$. These results remained the same in our sensitivity analysis, in which we excluded participants without MINI-diagnosed psychiatric comorbidity who were nevertheless prescribed psychotropic medication (data not shown).

The subgroup analysis revealed the influence of the specific psychiatric and substance use comorbidities on MMT treatment outcome (Table 4). Having a comorbid tranquilizer use disorder $(\beta=20.781 ; 95 \%$ confidence interval $[\mathrm{CI}]$ : 10.006, $31.555 ; P<0.001$; Table 4$)$ or cocaine use disorder $(\beta=6.344$;
Table 3 Influence of psychiatric comorbidity on percentage of opioid-positive urine drug screens $(\mathrm{N}=652)$

\begin{tabular}{llll}
\hline Covariates & Estimated $\beta$ & 95\% Cl & $P$-value \\
\hline Current age (years) & 0.173 & $-0.040,0.385$ & $0.1 \mathrm{II}$ \\
Sex & -2.542 & $-6.803,1.719$ & 0.242 \\
Methadone dose (mg/d)* & -0.074 & $-0.120,-0.028$ & 0.002 \\
Duration on MMT (months) & -0.035 & $-0.081,0.011$ & 0.139 \\
Psychiatric comorbidity & 3.069 & $-2.145,8.282$ & 0.248 \\
Using psychotropic medication & -2.084 & $-6.398,2.229$ & 0.343 \\
\hline
\end{tabular}

Notes: $R^{2}=0.026$, adjusted $R^{2}=0.017 . * P<0.05$.

Abbreviations: $\mathrm{Cl}$, confidence interval; $\mathrm{MMT}$, methadone maintenance treatment.

95\% CI: 0.592, 12.095; $P=0.031$; Table 4) was associated with continued illicit opioid use, with positive $\beta$-values indicating higher percentage of opioid-positive urine drug screens (Table 4). No other comorbid psychiatric disorders were significantly associated with the presence of opioidpositive urine drug screens (Table 4).

\section{Discussion}

\section{Implications for treatment}

In this large prospective study, we found a high proportion of patients with OUD to have other psychiatric disorders and SUDs, most commonly anxiety and mood disorders. The most commonly used substances in this population were cannabis and cocaine. Approximately two-thirds of patients in this study continued to use illicit opioids while receiving MMT.

MMT is not without risks, and the possibility of overdose and death is of key concern to clinicians. ${ }^{41,45,46}$ In particular, continued use of illicit opioids during methadone treatment carries significant risk of overdose.$^{47}$ Thus, urine screens

Table 4 Influence of specific psychiatric comorbidities on percentage of opioid-positive urine drug screens $(\mathrm{N}=652)$

\begin{tabular}{|c|c|c|c|}
\hline Covariates & Estimated $\beta$ & $95 \% \mathrm{Cl}$ & $P$-value \\
\hline Current age (years) & 0.161 & $-0.049,0.37 \mathrm{I}$ & 0.133 \\
\hline Sex & -1.533 & $-5.999,2.933$ & 0.500 \\
\hline Methadone dose $(\mathrm{mg} / \mathrm{d})^{*}$ & -0.060 & $-0.107,-0.014$ & 0.010 \\
\hline Duration on MMT (months) & -0.032 & $-0.077,0.014$ & 0.173 \\
\hline Mood disorder & -1.751 & $-6.144,2.643$ & 0.434 \\
\hline Anxiety disorder & -3.000 & $-7.426,1.427$ & 0.184 \\
\hline Psychotic disorder & -4.307 & $-16.483,7.868$ & 0.487 \\
\hline Antisocial personality disorder & 0.977 & $-4.220,6.175$ & 0.712 \\
\hline Alcohol use disorder & 0.303 & $-6.196,6.802$ & 0.927 \\
\hline Stimulant use disorder & -1.619 & $-10.53 \mid, 7.292$ & 0.721 \\
\hline Cocaine use disorder* & 6.344 & $0.592,12.095$ & 0.031 \\
\hline Hallucinogen use disorder & -4.528 & $-16.615,7.560$ & 0.462 \\
\hline Phencyclidine use disorder & 14.194 & $-7.795,36.184$ & 0.205 \\
\hline Cannabis use disorder & -0.701 & $-5.857,4.455$ & 0.790 \\
\hline Tranquilizer use disorder*** & 20.781 & $10.006,31.555$ & 0.000 \\
\hline Other & 10.146 & $-17.274,37.567$ & 0.468 \\
\hline
\end{tabular}

Notes: $R^{2}=0.074$, adjusted $R^{2}=0.05$. $* P<0.05$. $* * * P<0.001$.

Abbreviations: $\mathrm{Cl}$, confidence interval; $\mathrm{MMT}$, methadone maintenance treatment. 
for illicit opioids are an important measure of methadone treatment safety and efficacy.

We found that participants with comorbid tranquilizer and cocaine use disorders were more likely to show continued illicit opioid use while in MMT. The association with tranquilizer use disorder was particularly significant. Polysubstance use has been identified as a major contributing factor to decreased abstinence and retention in treatment, ${ }^{12}$ as well as methadone-related deaths, particularly early in the course of MMT. ${ }^{47,48}$ Cocaine, amphetamines, benzodiazepines, and alcohol are especially implicated in polysubstance use leading to death. ${ }^{49}$

In the MINI, "tranquilizers" are considered to include benzodiazepines, benzodiazepine-derivatives, barbiturates, and gamma-hydroxybutyrate. ${ }^{37}$ Benzodiazepines, in particular, are medications commonly prescribed for anxiety or insomnia that have well-known abuse liability ${ }^{50}$ and require careful consideration as to the risks associated with their use in MMT. ${ }^{51}$ Numerous studies have documented adverse effects of ongoing benzodiazepine use during MMT, negatively impacting abstinence, ${ }^{11}$ retention in treatment, ${ }^{12}$ and risk of relapse to opioid use..$^{52-54}$

An investigation by Chen et $\mathrm{a}^{55}$ revealed that half of the patients using benzodiazepines during MMT began using them, or increased use, upon commencing treatment for OUD. We found that approximately $25 \%$ of our study participants were taking routinely prescribed benzodiazepines - and there was no significant difference in rate of use between participants with and without MINI-diagnosed psychiatric comorbidity. Further, our study is in agreement with a study by White et $\mathrm{al},{ }^{56}$ which revealed that only nonprescription benzodiazepines were associated with negative consequences on MMT outcomes. We found that when participants reported tranquilizers as drugs of abuse, this was associated with continued illicit opioid use, whereas prescribed benzodiazepine use was not associated with the same (data not shown).

It has been shown that misuse of sedative medications, including benzodiazepines, is not improved by treatment in MMT. ${ }^{53}$ Understanding the reason for benzodiazepine misuse in MMT will be important for improving treatment outcomes. At the same time, treating anxiety or insomnia in patients who are dependent on opioids requires careful clinical consideration and recognizing which patients will benefit from benzodiazepine treatment for their psychiatric comorbidity is integral. ${ }^{57}$

In keeping with previous evidence, ${ }^{12,58,59}$ our study showed that cocaine use is also associated with continued opioid use while on MMT. Conversely, decreased cocaine use has been associated with cessation of illicit opioid use.${ }^{60}$ Cocaine use, however, does not appear to be significantly altered by treatment in MMT, ${ }^{53,61}$ and though occasional cocaine use may be reduced, there is little impact on regular use. ${ }^{62}$

Cannabis use disorder was the most prevalent substance use comorbidity we identified, though it had no association with continued illicit opioid use. Previous literature has been inconclusive regarding its impact on MMT outcome, with both beneficial ${ }^{63}$ and detrimental associations demonstrated ${ }^{64}$ As such, the association between cannabis use and MMT outcomes is an important area of ongoing study. ${ }^{65}$

Knowing factors associated with reduced abstinence from illicit opioids while on MMT is an opportunity for clinicians to improve outcomes and minimize serious adverse events such as drug-drug interactions, overdose, and mortality. Yet, knowledge of these risks, without appropriate intervention, does not suffice, as in many cases of methadone-related deaths, clinicians are aware of the patient's history of polysubstance abuse or dependence. ${ }^{48}$ The issue of comorbid substances is not uniquely addressed in MMT, and no specialized treatment plans for these patients are defined and implemented ${ }^{66}$ Our study adds to the knowledge of comorbid substance abuse during MMT and calls for vigilance in monitoring patients' drug use other than opioids.

Further, the presence of other comorbid psychiatric disorders must be acknowledged in MMT, and treatment should be tailored to maximize the likelihood of positive treatment outcomes. Approximately $80 \%$ of our participants in MMT met MINI criteria for one or more psychiatric disorders. Whether these patients had been previously recognized to have psychiatric comorbidity is unknown, and it is possible that without structured screening some patients may not receive appropriate diagnosis, treatment, and support. The high prevalence of psychiatric comorbidity in OUD suggests that a thorough review of past psychiatric history and current symptomatology is warranted for each patient entering MMT.

We found that many participants with no psychiatric comorbidity were regularly taking a psychotropic medication. For those patients with no identifiable psychiatric comorbidity, care must be taken to prevent polypharmacy and overmedication. On the other hand, it may be that these participants had a psychiatric disorder not captured by the MINI time frame for some disorders, or, alternatively, that the medications were managing their symptoms such that they were not captured on screening. Although these participants did not score to meet diagnostic criteria in the MINI, because they were prescribed psychotropic medications, they 
may have a psychiatric comorbidity. To account for this, we conducted a sensitivity analysis in which we excluded those participants from our main analysis and found no change in results: psychiatric comorbidity was not significantly associated with increased illicit opioid use while in MMT.

Despite finding no association between psychiatric comorbidity and abstinence from illicit opioid use, the impact on other MMT outcomes or quality of life measures was not evaluated. Psychiatric comorbidity in MMT patients has previously been associated with severely diminished quality of life. ${ }^{67}$ Improving appropriate diagnosis and treatment of comorbid psychiatric illness should meaningfully impact outcomes both in MMT and in other measures of well-being. Management of psychiatric comorbidity through on-site and integrated psychiatric care has been shown to improve psychiatric outcomes but may not impact abstinence or substance abuse outcomes. ${ }^{68}$

\section{Strengths and limitations}

To our knowledge, this is the largest study to date to detail the prevalence of comorbid psychiatric disorders and their influence on MMT outcome in patients receiving MMT for OUD.

Our study is strengthened by real-time diagnosis of psychiatric comorbidity, thus averting the need to use less reliable historical data in past medical records. However, a significant limitation associated with the MINI is the inability to distinguish current and lifetime comorbidity for each psychiatric disorder, as certain diagnoses reflect only 30-day symptomatology, whereas others are based on the presence of 12-month or lifetime symptoms. ${ }^{38}$ Therefore, it was not possible to distinguish current versus lifetime prevalence of psychiatric comorbidity in this study. We also lack data on the longitudinal progression of psychiatric symptomatology for each patient.

All data were collected from clinics in Southern Ontario, Canada. How well these results would generalize to other areas of the world must also be questioned. Finally, the average length of time in treatment for those with psychiatric comorbidity was 50.18 months, or greater than 4 years, thus the majority of patients had been receiving lengthy treatment. We have no data on patients who dropped out of MMT, however, which may result in a healthy adherer or compliance bias in our results. ${ }^{69,70}$ Future research should explore the association between psychiatric comorbidity and outcomes in patients newly entering MMT for OUD, as the findings may differ from our population that had already received lengthy treatment.

\section{Conclusion}

Psychiatric comorbidity, on the whole, was not significantly associated with MMT outcome, as evidenced by abstinence from illicit opioid use. However, comorbid cocaine and tranquilizer use disorders were significantly associated with increased use of illicit opioids. This study highlights a target population for whom more intensive treatment may be required to achieve optimal results in MMT for OUD.

\section{Acknowledgments}

The authors would like to acknowledge the contributions of Sheelagh Rutherford for her valuable assistance in data collection, the CATC staff and management team for their ongoing collaboration and facilitation of this study, The GENOA team members and volunteers for their countless hours and efforts that made this project possible. The authors would also like to acknowledge the significant contribution of the following study team members: Meha Bhatt, Laura Zielinski, Natalia Mouravska, and Guillaume Pare for their role in study design, acquisition of data, critically revising the manuscript, and approval of the manuscript final version; Jacqueline Hudson for data acquisition, entry, and quality management; Andrew Worster for critical revising the manuscript and approval of the final draft; Michael Varenbut for study design, data acquisition, approval of final draft, and accountability for all aspects of the study; Jeff Daiter for data acquisition and contribution to the study concept. The authors are most grateful to the study participants who volunteered their time, data, and efforts to complete this study that would not have been possible without their generosity. Tea Rosic is funded by the Mach-Gaensslen Foundation summer studentship. This work was supported by research grants from the Canadian Institute for Health Research (CIHR), Ottawa, ON, Canada, The Hamilton Academic Health Sciences Organization, Hamilton, ON, Canada, The Chanchlani Research Centre at the Population Genomics Program, Hamilton, ON, Canada, and Peter Boris Centre for Addictions Research, Hamilton, ON, Canada. The funding bodies have no role in the design of the study, the analysis of data, or publication of results.

\section{Disclosure}

The authors report no conflicts of interest in this work.

\section{References}

1. Degenhardt L, Whiteford HA, Ferrari AJ, et al. Global burden of disease attributable to illicit drug use and dependence: findings from the Global Burden of Disease Study 2010. Lancet. 2013;382(9904):1564-1574. 
2. Compton WM, Volkow ND. Major increases in opioid analgesic abuse in the United States: concerns and strategies. Drug Alcohol Depend. 2006;81(2):103-107.

3. Okie S. A flood of opioids, a rising tide of deaths. N Engl J Med. 2010; 363(21):1981-1985.

4. Degenhardt L, Whiteford H, Hall WD. The Global Burden of Disease projects: what have we learned about illicit drug use and dependence and their contribution to the global burden of disease? Drug Alcohol Rev. 2014;33(1):4-12.

5. Effective Medical Treatment of Opiate Addiction. National consensus development panel on effective medical treatment of opiate addiction. JAMA. 1998;280(22):1936-1943.

6. Mancino M, Curran G, Han C, Allee E, Humphreys K, Booth BM. Predictors of attrition from a national sample of methadone maintenance patients. Am J Drug Alcohol Abuse. 2010;36(3):155-160.

7. Deck D, Carlson MJ. Retention in publicly funded methadone maintenance treatment in two Western states. J Behav Health Serv Res. 2005;32(1):43-60.

8. Bell J, Burrell T, Indig D, Gilmour S. Cycling in and out of treatment: participation in methadone treatment in NSW, 1990-2002. Drug Alcohol Depend. 2006;81(1):55-61.

9. Brewer D, Catalano RF, Haggerty K, Gainey RR, Fleming CB. A metaanalysis of predictors of continued drug use during and after treatment for opiate addiction. Addiction. 1998;93(1):73-92.

10. Prendergast ML, Podus D, Chang E. Program factors and treatment outcomes in drug dependence treatment: an examination using metaanalysis. Subst Use Misuse. 2000;35(12-14):1931-1965.

11. Kamal F, Flavin S, Campbell F, Behan C, Fagan J, Smyth R. Factors affecting the outcome of methadone maintenance treatment in opiate dependence. Ir Med J. 2007;100(3):393-397.

12. Peles E, Schreiber S, Adelson M. Factors predicting retention in treatment: 10-year experience of a methadone maintenance treatment (MMT) clinic in Israel. Drug Alcohol Depend. 2006;82(3):211-217.

13. Villafranca SW, McKellar JD, Trafton JA, Humphreys K. Predictors of retention in methadone programs: a signal detection analysis. Drug Alcohol Depend. 2006;83(3):218-224.

14. Faggiono F, Vigna-Tagliantiu F, Versino E, Lemma P. Methadone maintenance at different dosages for opioid dependence. Cochrane Database Syst Rev. 2003;3:CD002208.

15. Naji L, Dennis B, Bawor M, et al. A prospective study to investigate predictors of relapse among patients with opioid use disorder treated with methadone. Subst Abuse. 2016;10:9-18.

16. Michna E, Ross EL, Hynes WL, et al. Predicting aberrant drug behavior in patients treated for chronic pain: importance of abuse history. J Pain Symptom Manage. 2004;28(3):250-258.

17. Martins SS, Keyes KM, Storr CL, Zhu H, Chilcoat HD. Pathways between nonmedical opioid use/dependence and psychiatric disorders: results from the National Epidemiologic Survey on Alcohol and Related Conditions. Drug Alcohol Depend. 2009;103(1-2):16-24.

18. Conway KP, Compton W, Stinson FS, Grant BF. Lifetime comorbidity of DSM-IV mood and anxiety disorders and specific drug use disorders: results from the National Epidemiologic Survey on Alcohol and Related Conditions. J Clin Psychiatry. 2006;67(2):247-257.

19. Strain EC. Assessment and treatment of comorbid psychiatric disorders in opioid-dependent patients. Clin J Pain. 2002;18(4 suppl): S14-S27.

20. Edlund MJ, Steffick D, Hudson T, Harris KM, Sullivan M. Risk factors for clinically recognized opioid abuse and dependence among veterans using opioids for chronic non-cancer pain. Pain. 2007;129(3): 355-362.

21. Rounsaville BJ, Kosten TR, Weissman MM, Kleber HD. Prognostic significance of psychopathology in treated opiate addicts. A 2.5-year follow-up study. Arch Gen Psychiatry. 1986;43(8):739-745.

22. Astals M, Diaz L, Domingo-Salvany A, Martin-Santos R, Bulbena A, Torrens M. Impact of co-occurring psychiatric disorders on retention in a methadone maintenance program: an 18-month follow-up study. Int J Environ Res Public Health. 2009;6(11):2822-2832.
23. Trafton JA, Minkel J, Humphreys K. Opioid substitution treatment reduces substance use equivalently in patients with and without posttraumatic stress disorder. J Stud Alcohol. 2006;67(2):228-235.

24. Cacciola JS, Alterman AI, Rutherford MJ, McKay JR, Mulvaney FD. The relationship between psychiatric comorbidity to treatment outcomes in methadone maintained patients. Drug Alcohol Depend. 2001; 61(3):271-280.

25. Rounsaville BJ, Weissman MM, Crits-Christoph K, Wilber C, Kleber H. Diagnosis and symptoms of depression in opiate addicts. Course and relationship to treatment outcome. Arch Gen Psychiatry. 1982;39(2):151-156.

26. Ouimette PC, Finney JW, Moos RH. Two-year posttreatment functioning and coping of substance abuse patients with posttraumatic stress disorder. Psychol Addict Behav. 1999;13:105-114.

27. King VL, Kidorf MS, Stoller KB, Carter JA, Brooner RK. Influence of antisocial personality subtypes on drug abuse treatment response. J Nerv Ment Dis. 2001;189(9):593-601.

28. Verthein U, Degkwitz P, Haasen C, Krausz M. Significance of comorbidity for the long-term course of opiate dependence. Eur Addict Res. 2005;11(1):15-21.

29. Ngo HT, Tait RJ, Hulse GK. Hospital psychiatric comorbidity and its role in heroin dependence treatment outcomes using naltrexone implant or methadone maintenance. J Psychopharmacol. 2011;25(6): 774-782.

30. Gelkopf M, Weizman T, Melamed Y, Adelson M, Bleich A. Does psychiatric comorbidity affect drug abuse treatment outcome? A prospective assessment of drug abuse, treatment tenure and infectious diseases in an Israeli methadone maintenance clinic. Isr J Psychiatry Relat Sci. 2006;43(2):126-136.

31. Samaan Z, Bawor M, Dennis BB, et al. Genetic influence on methadone treatment outcomes in patients undergoing methadone maintenance treatment for opioid addiction: a pilot study. Neuropsychiatr Dis Treat. 2014;10:1503-1508.

32. Bawor M, Dennis BB, Samaan MC, et al. Methadone induces testosterone suppression in patients with opioid addiction. Sci Rep. 2014;4:6189.

33. Dennis BB, Samaan MC, Bawor M, et al. Evaluation of clinical and inflammatory profile in opioid addiction patients with comorbid pain: results from a multicenter investigation. Neuropsychiatr Dis Treat. 2014;10:2239-2247.

34. Bawor M, Dennis BB, Varenbut M, et al. Sex differences in substance use, health, and social functioning among opioid users receiving methadone treatment: a multicenter cohort study. Biol Sex Differ. 2015;6:21.

35. von Elm E, Altman DG, Egger M, et al; STROBE Initiative. The Strengthening the Reporting of Observational Studies in Epidemiology (STROBE) statement: guidelines for reporting observational studies. Lancet. 2007;370(9596):1453-1457.

36. American Psychiatric Association. Diagnostic and Statistical Manual of Mental Disorders. 4th text rev ed. Washington DC: American Psychiatric Publishing; 2000.

37. Sheehan DV, Lecrubier Y, Sheehan KH, et al. The Mini-International Neuropsychiatric Interview (M.I.N.I.): the development and validation of a structured diagnostic psychiatric interview for DSM-IV and ICD-10. J Clin Psychiatry. 1998;59(suppl 20):22-33. quiz 34-57.

38. First MB, Spitzer RL, Gibbon M, Williams JB. Structured Clinical Interview for DSM-IV-TR Axis I Disorders, Research Version, Patient Edition. New York: (SCID-I/P) New York: Biometrics Research, New York State Psychiatric Institute; 2002.

39. Robins LN, Wing J, Wittchen HU, et al. The Composite International Diagnostic Interview. An epidemiologic Instrument suitable for use in conjunction with different diagnostic systems and in different cultures. Arch Gen Psychiatry. 1988;45(12):1069-1077.

40. NOVX Systems. inventor iMDx ${ }^{\mathrm{TM}}$. Available from: http://www. novxsystems.com/www.novxsystems.com/what-we-do/index.html. Accessed March 22, 2017. 
41. Mattick RP, Breen C, Kimber J, Davoli M. Methadone maintenance therapy versus no opioid replacement therapy for opioid dependence. Cochrane Database Syst Rev. 2009;3:CD002209.

42. Gossop M, Bradley B, Phillips GT. An investigation of withdrawal symptoms shown by opiate addicts during and subsequent to a 21-day in-patient methadone detoxification procedure. Addict Behav. 1987;12(1):1-6.

43. Wasserman DA, Korcha R, Havassy BE, Hall SM. Detection of illicit opioid and cocaine use in methadone maintenance treatment. Am J Drug Alcohol Abuse. 1999;25(3):561-571.

44. Peduzzi P, Concato J, Kemper E, Holford TR, Feinstein AR. A simulation study of the number of events per variable in logistic regression analysis. J Clin Epidemiol. 1996;49(12):1373-1379.

45. Cousins G, Teljeur C, Motterlini N, McCowan C, Dimitrov BD, Fahey T. Risk of drug-related mortality during periods of transition in methadone maintenance treatment: a cohort study. J Subst Abuse Treat. 2011;41(3):252-260.

46. Jimenez-Treviño L, Saiz PA, García-Portilla MP, et al. A 25-year follow-up of patients admitted to methadone treatment for the first time: mortality and gender differences. Addict Behav. 2011;36(12): 1184-1190.

47. Huang CL, Lee CW. Factors associated with mortality among heroin users after seeking treatment with methadone: a population-based cohort study in Taiwan. J Subst Abuse Treat. 2013;44(3):295-300.

48. Zador D, Sunjic S. Deaths in methadone maintenance treatment in New South Wales, Australia 1990-1995. Addiction. 2000;95(1):77-84

49. United Nations Office on Drugs and Crime. World Drug Report 2015. Herndon, VA: United Nations Publication; 2015. Sales No. E.15.XI.6.

50. O'brien CP. Benzodiazepine use, abuse, and dependence. J Clin Psychiatry. 2006;66(suppl 2):28-33.

51. Bleich A, Gelkopf M, Schmidt V, Hayward R, Bodner G, Adelson M. Correlates of benzodiazepine abuse in methadone maintenance treatment. A 1 year prospective study in an Israeli clinic. Addiction. 1999; 94(10):1533-1540.

52. Brands B, Blake J, Marsh DC, Sproule B, Jeyapalan R, Li S. The impact of benzodiazepine use on methadone maintenance treatment outcomes. J Addict Dis. 2008;27(3):37-48.

53. DeMaria PA Jr, Sterling R, Weinstein SP. The effect of stimulant and sedative use on treatment outcome of patients admitted to methadone maintenance treatment. Am J Addict. 2000;9(2):145-153.

54. Darke S, Ross J, Mills K, Teeson M, Williamson A, Havard A. Benzodiazepine use among heroin users: baseline use, current use and clinical outcome. Drug Alcohol Rev. 2010;29(3):250-255.

55. Chen KW, Berger CC, Forde DP, D'Adamo C, Weintraub E, Gandhi D. Benzodiazepine use and misuse among patients in a methadone program. BMC Psychiatry. 2011;11:90.
56. White WL, Campbell MD, Spence RD, Hoffman HA, Crissman B, DuPont RL. Patterns of abstinence or continued drug use among methadone maintenance patients and their relation to treatment retention. J Psychoactive Drugs. 2014;46(2):114-122.

57. Ciraulo DA, Nace EP. Benzodiazepine treatment of anxiety or insomnia in substance abuse patients. Am J Addict. 2000;9(4):276-279. Discussion 280-284

58. Proctor SL, Copeland AL, Kopak AM, Hoffmann NG, Herschman PL, Polukhina N. Predictors of patient retention in methadone maintenance treatment. Psychol Addict Behav. 2015;29(4):906-917.

59. Senbanio R, Wolff K, Marshall EJ, Strang J. Persistence of heroin use despite methadone maintenance treatment: poor coping self-efficacy predicts continued heroin use. Drug Alcohol Rev. 2009;28(6):608-615.

60. Maremmani I, Pani PP, Mellini A, et al. Alcohol and cocaine use and abuse among opioid addicts engaged in a methadone maintenance treatment program. J Addict Dis. 2007;26(1):61-70.

61. Hubbard RL, Craddock SG, Anderson J. Overview of 5-year followup outcomes in the drug abuse treatment outcome studies (DATOS). J Subst Abuse Treat. 2003;25(3):125-134.

62. Roux P, Lions C, Vilotitch A, et al. Correlates of cocaine use during methadone treatment: implications for screening and clinical management (ANRS Methaville study). Harm Reduct J. 2016;13:12.

63. Scavone JL, Sterling RC, Weinstein SP, Van Bockstaele EJ. Impact of cannabis use during stabilization on methadone maintenance treatment. Am J Addict. 2013;22(4):344-351.

64. Epstein DH, Preston KL. Does cannabis use predict poor outcome for heroin-dependent patients on maintenance treatment? A review of past findings, and more evidence against. Addiction. 2003;98(3):269-279.

65. Zielinski L, Bhatt M, Eisen R, et al. Association between cannabis use and treatment outcomes in patients receiving methadone maintenance treatment: a systematic review protocol. Syst Rev. 2016;5:139.

66. The College of Physicians and Surgeons of Ontario. Methadone Maintenance Treatment Program Standards and Clinical Guidelines. Toronto, ON: The College of Physicians and Surgeons of Ontario; 2011.

67. Carpentier PJ, Krabbe PF, van Gogh MT, Knapen LJ, Buitelaar JK, de Jong CA. Psychiatric comorbidity reduces quality of life in chronic methadone maintained patients. Am J Addict. 2009;18(6):470-480.

68. Brooner RK, Kidorf MS, King VL, et al. Managing psychiatric comorbidity within versus outside of methadone treatment settings: a randomized and controlled evaluation. Addiction. 2013;108(11):1942-1951.

69. Shrank WH, Patrick AR, Brookhart MA. Health user and related biases in observational studies of preventative interventions: a primer for physicians. J Gen Intern Med. 2001;26(5):546-550.

70. Delgado-Rodríguez M, Llorca J. Bias. J Epidemiol Community Health. 2004;58(8):635-641.

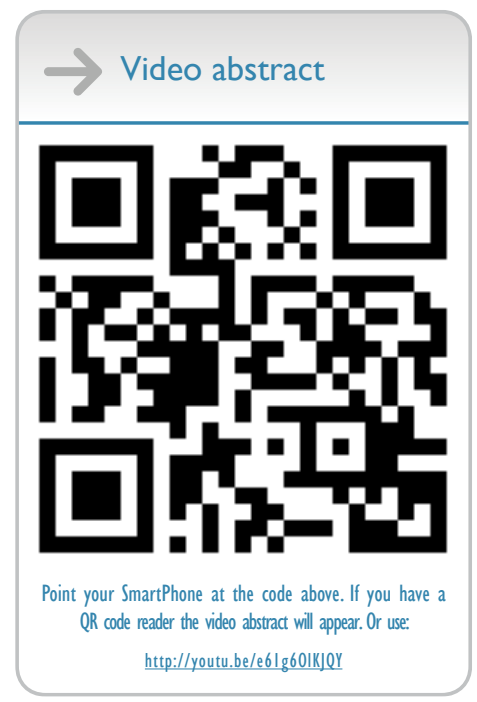

Neuropsychiatric Disease and Treatment 2017:13

submit your manuscript | www.dovepress.com 


\section{Publish your work in this journal}

Neuropsychiatric Disease and Treatment is an international, peerreviewed journal of clinical therapeutics and pharmacology focusing on concise rapid reporting of clinical or pre-clinical studies on a range of neuropsychiatric and neurological disorders. This journal is indexed on PubMed Central, the 'PsycINFO' database and CAS,

and is the official journal of The International Neuropsychiatric Association (INA). The manuscript management system is completely online and includes a very quick and fair peer-review system, which is all easy to use. Visit http://www.dovepress.com/testimonials.php to read real quotes from published authors.

Submit your manuscript here: http://www.dovepress.com/neuropsychiatric-disease-and-treatment-journal 